\title{
Sexualidade do Adolescente com deficiência: Ancoragens na literatura
}

\author{
Sexuality of adolescents with disabilities: Anchorages in literature
}

Sexualidad del Adolescente con discapacidad: Anclajes en la literatura

Rafaela Brandão Almeida Ambrosio ${ }^{1 *}$, Jorge Luís de Souza Riscado², Maria de Lourdes Fonseca Vieira², Fabrício de Medeiros Melo².

\section{RESUMO}

Objetivos: Investigar as publicações acerca da sexualidade do adolescente com deficiência considerando todos os tipos de deficiências e suas inter-relações e discutir a dificuldade na abordagem da temática. Métodos: Trata-se de uma revisão bibliográfica de cunho qualitativo em que a coleta de dados ocorreu entre janeiro e março de 2018, com a consulta de artigos indexados na Biblioteca Virtual em Saúde (BVS) publicados em periódicos no período de 2003 a 2018. Resultados: Observou-se a perpetuação de conceitos e práticas conservadoras que envolvem 0 assunto, sendo enfatizadas as infecções sexualmente transmissíveis ou questões biomédicas, com reduzida abordagem da temática sociocultural. Dentro dessa temática sociocultural, foram selecionados 13 artigos nos quais emergem como destaque o conflito do assunto para os pais, que em geral tendem a superproteger os filhos com deficiência; os valores familiares e sociais que tendem a negar a sexualidade entre adolescentes e a sexualidade do adolescente com deficiência, ainda estigmatizada como ausente ou como anormal, e com isso caminha-se para a negação o que potencializa e dificulta a abordagem da temática. Conclusões: A concretização da sexualidade do adolescente com deficiência necessita de uma ruptura de conceitos conservadores e estigmatizados. Na perspectiva da saúde, desde que seja tratada com respeito, pode proporcionar um melhor cuidar superando assim modelos supressores, permitindo um olhar para a inserção desse adolescente nesse mundo de descobertas e superando a subjetividade das práticas observadas nas inter-relações pessoais. Sendo necessário pensar em mecanismos que minimizem as barreiras existentes para o pleno desenvolvimento desses indivíduos. Palavras-Chave: Adolescente, Pessoa com deficiência, Sexualidade.

\footnotetext{
ABSTRACT

Objectives: To investigate the publications about the sexuality of adolescents with disabilities considering all types of disabilities and their interrelationships and discuss the difficulty in approaching the issue. Methods: This is a qualitative bibliographical review in which the data collection took place between January and March 2018, with the consultation of articles indexed in the Virtual Health Library (VHL) published in periodicals from 2003 to 2018. Results: The perpetuation of conservative concepts and practices involving the subject was observed, with emphasis on sexually transmitted infections or biomedical issues, with a reduced approach to sociocultural issues. Within this sociocultural theme, 13 articles were selected in which the conflict of the subject emerges as a highlight for parents, who in general tend to overprotect children with disabilities; the family and social values that tend to deny the sexuality between adolescents and the sexuality of the adolescent with disability, still stigmatized as absent or abnormal, and with this goes to the negation which

${ }^{1}$ Faculdade de Medicina do Centro de Estudos Superiores de Maceió, Maceió-AL.

${ }^{2}$ Faculdade de Medicina da Universidade Federal de Alagoas, Maceió-AL.

* E-mail: rafaelabrandaoalmeida@gmail.com
} 
potentiates and makes difficult to approach the subject. Conclusions: Achieving the sexuality of adolescents with disabilities needs a rupture of conservative and stigmatized concepts. From the point of view of health, as long as it is treated with respect, it can provide a better care by overcoming suppressive models, allowing a look at the insertion of this adolescent in this world of discoveries and overcoming the subjectivity of the practices observed in the personal relationships. It is necessary to think of mechanisms that minimize the existing barriers to the full development of these individuals.

Keywords: Adolescent, Disabled person, Sexuality.

\section{RESUMEN}

Objetivos: Investigar las publicaciones acerca de la sexualidad del adolescente con discapacidad considerando todos los tipos de deficiencias y sus interrelaciones y discutir la dificultad en el abordaje de la temática. Métodos: Se trata de una revisión bibliográfica de cuño cualitativo en que la recolección de datos ocurrió entre enero y marzo de 2018, con la consulta de artículos indexados en la Biblioteca Virtual en Salud (BVS) publicados en periódicos en el período de 2003 a 2018. Resultados: Se observó la perpetuación de conceptos y prácticas conservadoras que envuelven el asunto, siendo enfatizadas las infecciones sexualmente transmisibles o cuestiones biomédicas, con reducido abordaje de la temática sociocultural. Dentro de esta temática sociocultural, se seleccionaron 13 artículos en los que emergen como destaque el conflicto del asunto para los padres, que en general tienden a sobreproteger a los hijos con discapacidad; los valores familiares y sociales que tienden a negar la sexualidad entre adolescentes y la sexualidad del adolescente con discapacidad, todavía estigmatizada como ausente o como anormal, y con ello se camina hacia la negación lo que potencia y dificulta el abordaje de la temática. Conclusiones: La concreción de la sexualidad del adolescente con discapacidad necesita una ruptura de conceptos conservadores y estigmatizados. En la perspectiva de la salud, desde que sea tratada con respeto, puede proporcionar un mejor cuidado superando así modelos supresores, permitiendo una mirada a la inserción de ese adolescente en ese mundo de descubrimientos y superando la subjetividad de las prácticas observadas en las interrelaciones personales. Es necesario pensar en mecanismos que minimicen las barreras existentes para el pleno desarrollo de esos individuos.

Palabras clave: Adolescente, Persona con discapacidad, La sexualidade.

\section{INTRODUÇÃO}

A sexualidade é consequência da experiência das pessoas em seu contexto geral, resultado da interação de fatores biológicos, psicológicos, sócios econômicos, culturais, éticos e religiosos e está ligada ao desenvolvimento global do indivíduo, constituindo um dos elementos de sua personalidade (GLAT, 2004; NUNES, 2005).

É a maneira de construir, aprender, ser, compreender e viver o mundo e de se expressa por meio da integração, corpo e mente, que se reflete como base para a feminilidade ou masculinidade do indivíduo sendo um ingrediente do desenvolvimento da identidade do individuo com reflexo nas relações física e mental. (OZORIO, 1992; BRETAS, 2004; GLAT, 2004) Manifesta-se no ser humano independe de se ter ou não uma deficiência (MAIA, 2001) sendo a forma dessa expressão também variada independente de ser uma pessoas com deficiência ou não-deficientes, a sexualidade seus sentimentos e comportamentos terão diversas formas de se apresentar e quando bem conduzida, coopera para o bom desenvolvimento da afetividade e das relações interpessoais, o que contribui para uma melhor valorização de si mesmo e em adaptação à sociedade (GEJER, 2001; KAUFMAN, et al., 2003; GIAMI, 2004; MAIA, 2006). 
Uma boa evolução da sexualidade para crianças e adolescentes tem sua ancoragem e evolução pautada nos relacionamentos, no equilíbrio emocional e na manifestação de sentimentos sendo ainda influenciada por questões de familiares, fatores morais, culturais, sociais, religiosos (COSTA et al.,2001). Destacando que a sexualidade envolve para além da descoberta do outro a descoberta de si que pode realizar-se de várias formas sejam sensorial, cognitiva de linguagem que se apresentam como elemento de formação dessa identidade (ROMERO et al., 2007; MATOS et al., 2005; CANO et al.,2000; DAVIM et al., 2008).

Para Mazzota e D'Antino (2011), as circunstâncias em que se inserem as pessoas com os vários tipos de deficiência tanto de ordem pessoal quanto ambiental podem tanto favorecer quanto criar e ampliar desvantagens para pessoas com deficiências (MAZZOTA e D'ANTINO, 2011).

O senso comum difunde e expressa um estigma sobre a sexualidade dos adolescentes com deficiência, necessitando assim que os aspectos de crescimento e desenvolvimento biopsicossocial e sexual alcancem ampla e adequada proporções, evitando que esses adolescentes se tornem expostos a riscos, liberdades e responsabilidades, sem o devido preparo (LEITE et. al., 2007)

Importantes recomendações internacionais, contemplado a Cultura, a Educação, o Lazer e demais setores sociais e voltadas a diversidade da condição humana já foram incorporadas à legislação brasileira. Entretanto como já destacam Mazzota e D'Antino (2011) há enorme descompasso entre aquilo que se pensa e deseja e o que de fato é transformado (MAZZOTA e D'ANTINO, 2011).

A Política Nacional de Saúde da Pessoa com Deficiência instituída pelo Ministério da Saúde, por meio da Portaria MS/GM № 1.060, de 5 de junho de 2002 garante a inclusão das pessoas com deficiência em todos os âmbitos considerando-as como cidadãs iguais em direitos e diferentes em necessidades, que devem ser supridas pelo Estado, por meio de políticas públicas e, solidariamente, pela sociedade como um todo (BRASIL, 2002).

A adolescência é um período de transição, em busca da independência e identidade adulta. A puberdade e o desenvolvimento das características sexuais primárias e secundárias são considerados o fenômeno desencadeador das grandes mudanças e transformações que caracterizam esse período da vida. (CAMARGO, et. al., 1994; GEJER e KLOURI, 2002).

A discussão do tema sexualidade em nossa cultura ainda surge acompanhada de preconceito. Quando esse tema é voltado para a pessoa com deficiência, essas constatações tornam-se ainda mais reais (BRASIL, 2009).

Para a pessoa com deficiência a sexualidade não deve ser considerada isoladamente, mas dentro de um contexto global da vida, e quando bem conduzida é decisiva na implementação do desenvolvimento afetivo e da capacidade de estabelecer contatos interpessoais, fortalecendo a autoestima, o bem-estar, o amor próprio e a adequação à sociedade (GEJER e KLOURI, 2002; COSTA, et al., 2001).

Conod e Servais (2008) observaram que os fatores sócio-culturais e pessoais devem ser considerados quando se analisa questões de sexualidade de pessoas com deficiência, identificando como questões fundamentais a serem abordadas as relacionadas as áreas de contracepção, gestão da higiene, educação sexual e prevenção de ITS ou estupro (CONOD e SERVAIS, 2008).

As atitudes sociais e as percepções são resultadas de uma construção do conhecimento assim ESMAIL et al. (2010) sugere que há pouca exposição da sociedade quando associado a sexualidade e a deficiência, o que se reflete em uma sociedade ainda sem muita aproximação com o tema (ESMAIL, et al., 2010). 
A Convenção dos Direitos da Criança (ONU/1989) reforça o direito de acesso e acessibilidade das crianças com deficiência aos serviços de educação e saúde, o que deverá incluir as questões referentes à educação em sexualidade e aos direitos sexuais e reprodutivos. Tema ainda considerado tabu para ser abordado com crianças, sejam elas com deficiência ou não.

Assim, faz-se necessário instrumentalizar profissionais que trabalham com as pessoas com deficiência, que devem transmitir esses conceitos ao adolescente com deficiência e sua família, em um aspecto de preparação para a vida adulta. É urgente que propostas de orientação sexual sejam colocadas em prática como parte dos currículos educacionais junto à população especial (MAIA e CAMOSSA, 2003).

A partir dessa perspectiva, objetiva-se analisar a literatura específica acerca da sexualidade do adolescente com deficiência sem especificar ou diferenciar o tipo de deficiência. Espera-se estabelecer hipóteses ou pressupostos, a fim de nortear o desenvolvimento de projetos como investigações e intervenções acerca do assunto.

\section{METODOLOGIA}

Neste estudo realizamos uma pesquisa de revisão de literatura, aqui entendida como um estudo exploratório da produção do conhecimento acerca de um assunto ou tema, verificando assim, o conhecimento produzido e obtendo elementos para analisá-lo. Como fonte de dados foram consultados artigos científicos indexados nas bases eletrônicas LILACS, SCIELO, MEDLINE publicados em periódicos, no período de 2003 a 2018

A pesquisa bibliográfica ocorreu no período de janeiro a março de 2018, e incluiu estudos dos últimos 15 anos que analisavam a temática da sexualidade do adolescente com deficiência com o foco na perspectiva sociocultural, assim foram incluídos artigos científicos completos, gratuitos e em pdf, e excluídos publicações como teses de doutorado e dissertações de mestrado e artigos de revisão de literatura bem como artigos que se voltavam particularmente para as questões genética e biomédica.

Os descritores utilizados para a busca nas bases de dados foram: Sexualidade; Pessoa com Deficiência e Adolescente presentes no titulo, resumo ou palavra-chave. Resultando em 201 publicações identificadas das quais, depois de aplicados os critérios de exclusão resultaram em 13 artigos elegíveis.

Destacamos que as generalizações devem ser ponderadas e evitadas, pois nos utilizamos para análise de uma biblioteca específica e assim estamos discutindo a partir das perspectivas, dos achados e posicionamentos desses autores.

Após a leitura e análise crítica dos artigos, as publicações foram caracterizar quanto ao ano de publicação, país de origem do estudo, objetivo central da discussão e método utilizado. A fim de facilitar a visualização esse resultado foi consolidado e agrupado em um quadro (Quadro 1).

Em seguida utilizou-se de uma adaptação, da técnica de análise de conteúdo, modalidade temática, descrita por Bardin (1979) que utiliza o tema como conceito central. Após a leitura central dos artigos foi identificada as ideias relacionadas a sexualidade da pessoa com deficiência, em seguida realizada a classificação das ideias em temas que resumem o conhecimento acerca do assunto estudado resultando na elaboração de sínteses interpretativas de cada tema. 


\section{RESULTADOS E DISCUSSÃO}

\section{Caracterização}

O presente artigo tem como foco de abordagem a questão sociocultural da deficiência, verificada em apenas 13 estudos identificados a partir dos descritores: Adolescente, Pessoa com deficiência e Sexualidade.

Quadro 1. Caracterização dos estudos analisados

\begin{tabular}{|c|c|c|c|c|c|}
\hline Estudo & Ano & País & Foco & Objetivo & Método \\
\hline $\begin{array}{l}\text { SCHLIEMANN et } \\
\text { al., }\end{array}$ & 2005 & BRASIL & $\begin{array}{l}\text { Sexualidade e } \\
\text { Deficiência } \\
\text { mental }\end{array}$ & $\begin{array}{l}\text { Entender como se dá a } \\
\text { informação e a vivência do } \\
\text { ensino da sexualidade na } \\
\text { adolescência de deficientes } \\
\text { portadores de deficiência mental. }\end{array}$ & $\begin{array}{l}\text { Estudo com } \\
\text { entrevista } \\
\text { semiestruturada }\end{array}$ \\
\hline LEME e CRUZ & 2008 & BRASIL & $\begin{array}{l}\text { Sexualidade do } \\
\text { adolescente } \\
\text { com Síndrome } \\
\text { de Down }\end{array}$ & $\begin{array}{l}\text { Identificar os adolescentes com } \\
\text { SD envolvendo dados } \\
\text { demográficos e da sexualidade, } \\
\text { segundo a concepção de seus } \\
\text { pais }\end{array}$ & $\begin{array}{l}\text { Estudo com } \\
\text { entrevista } \\
\text { semiestruturada }\end{array}$ \\
\hline LITTIG & 2012 & BRASIL & $\begin{array}{l}\text { Sexualidade na } \\
\text { deficiência } \\
\text { intelectual }\end{array}$ & $\begin{array}{l}\text { Investigar as concepções que } \\
\text { mães de jovens com deficiência } \\
\text { intelectual têm sobre a } \\
\text { sexualidade deles e como elas } \\
\text { irão refletir na adoção de práticas } \\
\text { de educação sexual }\end{array}$ & $\begin{array}{l}\text { Estudo com } \\
\text { entrevista } \\
\text { semiestruturada }\end{array}$ \\
\hline ALBUQUERQUE & 2011 & BRASIL & $\begin{array}{l}\text { Sexualidade e } \\
\text { Deficiência } \\
\text { mental }\end{array}$ & $\begin{array}{l}\text { Descrever as atitudes de pais e } \\
\text { de profissionais face a } \\
\text { sexualidade da pessoa com } \\
\text { deficiência mental. }\end{array}$ & $\begin{array}{l}\text { Estudo com } \\
\text { entrevista } \\
\text { semiestruturada }\end{array}$ \\
\hline $\begin{array}{l}\text { SANTOS } \\
\text { ÓSORIO }\end{array}$ & 2010 & BRASIL & $\begin{array}{l}\text { Sexualidade e } \\
\text { Deficiência } \\
\text { mental }\end{array}$ & $\begin{array}{l}\text { Evidenciar a relação existente } \\
\text { entre a produção do saber e as } \\
\text { práticas } \\
\text { cotidianas que se estabelecem } \\
\text { no meio social }\end{array}$ & $\begin{array}{l}\text { Pesquisa } \\
\text { documental }\end{array}$ \\
\hline MAIA e CAMOSA & 2003 & BRASIL & $\begin{array}{l}\text { Sexualidade e } \\
\text { Deficiência } \\
\text { mental }\end{array}$ & $\begin{array}{l}\text { Obter e analisar relatos sobre } \\
\text { sexualidade de cinco jovens, de } \\
\text { ambos os sexos, com deficiência } \\
\text { mental. }\end{array}$ & $\begin{array}{l}\text { Estudo com } \\
\text { entrevista } \\
\text { individual, } \\
\text { Desenho e Relato } \\
\text { Verbal }\end{array}$ \\
\hline LUIZ e KUBO & 2007 & BRASIL & \begin{tabular}{lr}
\multicolumn{2}{l}{ Relacionamento } \\
amorosos & para \\
jovens & com \\
Síndrome & de \\
Down & \\
\end{tabular} & $\begin{array}{l}\text { Descobrir quais as percepções } \\
\text { de jovens com Síndrome de } \\
\text { Down sobre relacionar-se } \\
\text { amorosamente. }\end{array}$ & $\begin{array}{l}\text { Estudo com } \\
\text { entrevista } \\
\text { semiestruturada }\end{array}$ \\
\hline MOURA e PEDRO & 2005 & BRASIL & $\begin{array}{l}\text { Sexualidade e } \\
\text { Deficiência } \\
\text { visual }\end{array}$ & $\begin{array}{l}\text { Conhecer as percepções dos } \\
\text { adolescentes portadores de } \\
\text { deficiência visual acerca de sua } \\
\text { sexualidade. }\end{array}$ & $\begin{array}{l}\text { Estudo com } \\
\text { entrevista } \\
\text { semiestruturada }\end{array}$ \\
\hline $\begin{array}{l}\text { BEZERRA } \\
\text { PAGLIUCA }\end{array}$ & 2010 & BRASIL & $\begin{array}{l}\text { Sexualidade e } \\
\text { Deficiência } \\
\text { visual }\end{array}$ & $\begin{array}{l}\text { Identificar a opinião das } \\
\text { adolescentes deficientes visuais } \\
\text { acerca da sua sexualidade. }\end{array}$ & $\begin{array}{l}\text { Estudo com } \\
\text { entrevista } \\
\text { semiestruturada }\end{array}$ \\
\hline $\begin{array}{l}\text { BASTOS } \\
\text { DESLANDES }\end{array}$ & 2012 & BRASIL & $\begin{array}{l}\text { Deferência } \\
\text { intelectual para } \\
\text { pais de } \\
\text { adolescentes. }\end{array}$ & $\begin{array}{l}\text { Investigar as narrativas sobre a } \\
\text { sexualidade de adolescentes } \\
\text { com deficiência intelectual, a } \\
\text { partir do relato de seus } \\
\text { responsáveis, e também } \\
\text { conhecer como esses } \\
\text { cuidadores declararam } \\
\text { comportar-se diante da } \\
\text { expressão sexual dos filhos. }\end{array}$ & $\begin{array}{l}\text { Análise } \\
\text { narrativas }\end{array}$ \\
\hline
\end{tabular}




\begin{tabular}{|c|c|c|c|c|c|c|}
\hline $\begin{array}{l}\text { SOARES, } \\
\text { MOREIRA } \\
\text { MONTEIRO }\end{array}$ & e & 2008 & $\begin{array}{l}\text { BRASIL } \\
\text { e EUA }\end{array}$ & $\begin{array}{l}\text { Sexualidade e } \\
\text { Deficiência } \\
\text { física }\end{array}$ & $\begin{array}{l}\text { Discutir a qualidade da vida de } \\
\text { jovens portadores de espinha } \\
\text { bífida em duas culturas: } \\
\text { brasileira e norte-americana. } \\
\text { Observando a percepção, } \\
\text { interesse e problematização dos } \\
\text { participantes em relação à sua } \\
\text { sexualidade e seus } \\
\text { desdobramentos. }\end{array}$ & $\begin{array}{l}\text { Estudo com } \\
\text { entrevista } \\
\text { semiestruturada } \\
\text { e grupo focal }\end{array}$ \\
\hline ESMAIL, et al. & & 2010 & CANADÁ & $\begin{array}{l}\text { Sexualidade e } \\
\text { Deficiência } \\
\text { percepção } \\
\text { social }\end{array}$ & $\begin{array}{l}\text { Identificar a percepção social da } \\
\text { sexualidade na deficiência. }\end{array}$ & $\begin{array}{ll}\text { Estudo } & \text { com } \\
\text { grupo focal }\end{array}$ \\
\hline $\begin{array}{l}\text { MORALES } \\
\text { BATISTA }\end{array}$ & e & 2010 & BRASIL & $\begin{array}{l}\text { Sexualidade na } \\
\text { deficiência } \\
\text { intelectual }\end{array}$ & $\begin{array}{l}\text { Descrever a compreensão de } \\
\text { conceitos sobre sexualidade por } \\
\text { jovens com diagnóstico de } \\
\text { deficiência intelectual, em aulas } \\
\text { de orientação sexual. }\end{array}$ & $\begin{array}{l}\text { Viodegração de } \\
\text { aulas }\end{array}$ \\
\hline
\end{tabular}

Fonte: Estudos analisados, 2018.

Os estudos selecionados foram realizados em sua maioria (onze) exclusivamente no Brasil e encontramse pulverizados por todo o período estudado. Tendo a maioria como foco central a sexualidade na percepção dos pais ou da própria pessoa com deficiência e voltada para uma deficiência específica, em sua maioria a deficiência intelectual.

Quanto ao percurso metodológico todos se ancoram na abordagem qualitativa oriunda, principalmente, das ciências sociais aplicadas à saúde permitindo a associação de características e significados aos valores do sujeito.

\section{A tematização da sexualidade do adolescente com deficiência - Sexualidade da pessoa com deficiência: conflito para os pais.}

Alguns jovens com deficiência são privados de informação, contato social e até modelos interessantes de relações afetivas (SCHLIEMANN, 2005). Alguns autores destacam a angustia o medo e a falta de informação como apontadas por pais e responsáveis de pessoas com deficiência como principais dificuldades em educar para a sexualidade seus filhos (SCHLIEMANN, 2005; LUIZ e KUBO, 2007; BASTSOS e DESLANDES, 2009; BEZERRA e PAGLIUCA, 2010; LITTIG, 2012).

Essa dificuldade é bem observada quando se identifica que os pais não encontram dificuldade na mesma proporção quando os filhos são ditos de desenvolvimento padrão (SCHLIEMANN, 2005). Destacando assim a necessidade de um olhar mais direcionado para esses pais e responsáveis para que essa lacuna seja superada e eles entendam a importância de que o tema seja tratado com seus filhos com deficiência bem como para que se sintam seguros para essa atribuição.

Quando presente a educação quanto a sexualidade a filhos com deficiências tem uma tendência a se restringir a aspectos genitais (SCHLIEMANN, 2005; LEME e CRUZ, 2008; ALBUQUERQUE, 2011). Albuquerque (2011) destaca que o uso de técnicas adequadas de educação sexual focada nos pais pode favorecer a compreensão da sexualidade dos filhos com deficiência e a abordagem da questão de forma mais adequada (ALBUQUERQUE, 2011).

Bastos e Deslandes (2012) identificaram em seu estudo uma dificuldade entre os pais de adolescentes com deficiência por não conseguirem enxergá-los com maturidade sexual e por não saberem como se comportar, principalmente, quando os filhos manifestavam a genitalidade, assim a negação, a repressão, e 
infantilização são com frequência o caminho adotado para mascarar a realidade que se apresenta e assim não visualizar a necessidade que o filho apresenta (BASTOS e DESLANDES, 2012; VIVARTA, 2003).

Em seu estudo Littig (2012) observou que as mães veem seus filhos com deficiência com pouca ou nenhuma possibilidade de crescimento pessoal e muito menos de desenvolvimento de interesses e comportamentos afetivo sexual e assim reproduzem a concepção social vigente em nossa cultura que nega e rejeita a existência da sexualidade (LITTIG, 2012).

Leme e Cruz (2008) observaram em seu estudo que mesmo os pais ou responsáveis que consideram a sexualidade existente, em seus filhos com deficiência, destacam que ela deve ser vivida e comedida com limites impostos pelos pais e demonstraram dificuldade em aceitar as expressões dessa sexualidade acreditando na perspectiva de o que se não estimulada ela pode ser contida. Assim muitas vezes quando a orientação parental existe tende a ser impessoal, difusa e geralmente restrita as questões ligadas ao órgão genital (BEZERRA e PAGLIUCA, 2010).

Bastos e Deslandes (2012) observaram que muitos pais acreditavam no estigma de que a sexualidade está ligada exclusivamente ao sexo a assim entendem que seus filhos se revestem somente de afetividade, separando da genitalidade, desqualificando a relação entre afetividade e sexualidade bem como a amplo conceito desta, fundamental e singular na construção identidade de cada individuo (BASTOS e DESLANDES, 2012).

Entretanto Littig (2012) ressalta que é extensivo a pessoa com deficiência a importância de que a família estabeleça uma relação de confiança e uma abertura para falar sobre sexualidade com seus adolescentes respeitando limites e possibilidade estabelecendo um discurso adequado a cada realidade e contemplando a saúde, os afetos e o prazer (LITTIG, 2012).

Uma vez que a mudança envolve valores pessoais construídos durante a vida, o tema é de abordagem ainda mais difícil. A dificuldade dos pais é um estimulo para que optem por ignora-lo e eternizar seus filhos como crianças com a ideia de que ao ocultar o tema ele não fará parte da vida de seus filhos (SCHLIEMANN, 2005; ALBUQUERQUE, 2011).

Assim os profissionais que assistem a esses adolescentes necessitam estar preparados para empoderar os pais a fim de anular ideias equivocadas, construir estratégias adequadas e promover a inclusão, para que que seus filhos possam viver plena e adequadamente sua sexualidade sem reproduzir a tendência da negação e contribuindo para a desmistificação (LUIZ e KUBO, 2007; ALBUQUERQUE, 2011). Isso a partir de estratégias que atendam as necessidades desse publico de forma personalizada.

\section{Valores familiares e sociais e a sexualidade da pessoa com deficiência}

Receios, preconceitos, valores culturais e religiosos são também apontados por alguns autores como entraves para um desenvolvimento pleno dos adolescentes com deficiência quanto a sexualidade (LEME e CRUZ, 2008; ALBUQUERQUE, 2011, LITTIG, 2012). O receio da forma como o tema vai alcançar seus filhos e a fim de que não fuja de seu controle, esses valores são ainda tidos como impeditivos para os pais aceitem a perspectiva de que o tema seja discutido por outra instituição que não a família (LEME e CRUZ, 2008).

O próprio preconceito, tabus e indefinições e mesmo a perspectiva de terem que refletir sobre os aspectos comedidos da própria sexualidade é sufocante e assim ainda preditivo para que muitas mães com a ideia de resguardá-los busquem justificativa para evitar o contato social e intimo de seus filhos (LITTIG, 2012). Evitando a necessidade de refletir sobre o tema, resguardando os próprios conflitos e preservando a vergonha que envolve a temática. 
Para Esmail et al. (2010) os indivíduos com deficiência são geralmente vistos como assexuados devido a uma ideia de sexualidade vinculada exclusivamente ao sexo o que contribui para a não discussão do assunto, culminando com a falta de informação, educação e dialogo sobre sexualidade e deficiência que é considerada um dos principais fatores que contribuem para a manutenção do estigma quando associados deficiência e sexualidade e assim mantendo-se o circulo vicioso (ESMAIL et al., 2010).

Bastos e Deslandes (2005) enfatizam que os adolescentes com deficiência possuem necessidades, impulsos, desejos e emoções normais, vivenciam e manifestam a sexualidade como quaisquer outros jovens e assim também necessitam que a resistência seja quebrada e respeitando as adaptações e individualidades a temática esteja em pauta dentro dessas famílias (BASTOS e DESLANDES, 2005).

Em sua maioria os adolescentes com deficiência são capazes de alcançar algum nível de autonomia, desde que para isso sejam apresentados a essa possibilidade da forma individualizada e adequada as necessidades (BASTOS e DESLANDES, 2012). Respeitando sua deficiência bem como sua realidade cultural, social e religiosa, direcionando uma abordagem adequada.

Assim em uma busca de superar essa resistência dos pais ALBUQUERQUE (2011) sugere que o foco das abordagens quanto a sexualidade da pessoa com deficiência deva ser a prevenção. Bastos e Deslandes (2007) defendem que o contato com o outro é fundamental na construção da autonomia desses indivíduos (BASTOS e DESLANDES, 2012).

Albuquerque (2011) Observou que pais que participam de cursos de capacitação quanto a sexualidade apresentam verbalizações que indicam o aumento da percepção acerca dos interesses afetivo-sexuais por parte dos filhos, apresentando indicações de aceitação e reconhecimento do direito do filho em exercer sua sexualidade (ALBUQUERQUE, 2011).

Bastos e Deslandes (2012) observaram em seu estudo que o meio social em que vivem as Pessoas com Deficiência - PCD, com influência de valores religiosos, de forma geral, é relevante a presença de muitos preconceitos em questões relacionadas à sexualidade. Observaram ainda que a existência da aceitação das expressões da sexualidade masculina e negação das do gênero oposto (BASTOS; DESLANDES, 2012).

Para Luiz e Kubo (2007) a naturalidade em abordar a sexualidade da pessoa com deficiência será um reflexo do reconhecimento de que esses indivíduos são capazes de tornarem-se participantes da vida social como outra pessoa qualquer (LUIZ e KUBO, 2007).

O estigma pode levar os indivíduos a internalizar conceitos de assexualidade e pode impactar negativamente na confiança, e no desejo, distorcendo a noção de sexualidade (ESMAIL, et al., 2010) e assim em um ciclo vicioso mante-se a perspectiva da negação fortemente marcada por valores familiares e sociais.

\section{O adolescente com deficiência e sua sexualidade}

Acredita-se que as pessoas, mesmo as com deficiência devem e poder ter suas próprias decisões e conhecimentos sobre sua saúde, exercendo assim seus direitos e deveres para o pleno exercício de sua cidadania (MOURA; PEDRO, 2006). Respeitando a importância dos sentimentos, das emoções e o propicio dentro da conjuntura da deficiência, cada individuo um pode e deve viver bem sua sexualidade (DE PAULA, et al., 2005).

Entretanto há evidências que constatam que ainda existe a visão mítica de que os jovens com deficiência não são capazes de compreender e exercer sua sexualidade (MORALES e BATISTA, 2010). Aferindo a 
perspectiva de que são assexuadas, noções pautadas pela infantilização desse grupo social, bem como pela perspectiva da sexualidade voltada exclusivamente para as relações de procriação.

Em geral as informações envolvendo a sexualidade repassadas a esses adolescentes se restringe a falar sobre os órgãos genitais e normas de convivência em publico (SCHLIEMANN, 2005; ALBUQUERQUE, 2011; LITTIG, 2012). Assim os adolescentes com deficiência têm informações limitadas quanto a sexualidade, e quando ela existe geralmente é advinda da informação que circula na sociedade, cercada de mitos e não tendo uma informação direcionada (BEZERRA e PAGLIUCA, 2010), o que com frequência estabelece o medo para esses adolescentes quando se trata da sexualidade.

Atitudes sociais e percepções são movidas por educação e conhecimento, se não houver uma ampla divulgação da existência de relação entre sexualidade e deficiência, fica difícil que a se tenha um entendimento estreito dessas questões (ESMAIL, et al., 2010).

Muitas vezes os pais desses adolescentes em busca de aceitação estimulam que eles, para que sejam aceitos, se tornarem extremamente carinhosos (SCHLIEMANN, 2005). DE PAULA et al. (2005) destaca que a afetividade é parte da sexualidade e se relaciona à capacidade de entrar em contato consigo e com o outro, o que é necessário e indispensável para o desenvolvimento da autoestima (DE PAULA, et al., 2005).

Quando um jovem com deficiência demonstra sua sexualidade de maneira inapropriada, em público ou de maneira compulsiva, há indícios de que possui poucas oportunidades de socialização e de obtenção de prazer (LEME e CRUZ, 2008). Para superar essa realidade que muitas vezes reflete em uma percepção errônea e estereotipada pela sociedade de uma sexualidade exacerbada entre as pessoas com deficiência Littig (2012) destaca que esses adolescentes precisam ser educados sexualmente como no caso de qualquer outra pessoa sem deficiência através do desenvolvimento de suas habilidades e competências com maior independência permitindo um real processo de inclusão (LITTIG, 2012).

Nessa perspectiva faz-se necessário valorizar o desenvolvimento, as crenças, os desejos, as experiências, a auto percepção e as relações sociais particulares de cada adolescente e de sua deficiência permitindo um avanço mais significativo e amplo na temática (SOARES, et al., 2008).

\section{Dificuldade de acesso a informações}

A escassez de recursos, treinamento e infraestrutura para orientar cuidadores e pacientes no atendimento de necessidades sexuais contribuem para a dificuldade de acesso a informação entre as pessoas com deficiência. Nesse cenário Conod e Servais (2008) destaca que uma grande questão se apresenta: como os profissionais de saúde, os cuidadores e os pais podem ajudar as pessoas com deficiência a viver a sua própria sexualidade (CONOD e SERVAIS, 2008).

Para Soares (2008) o déficit de informações está presente tanto em alguns serviços de reabilitação como, principalmente, na atenção básica em saúde. Destacando que tanto na formação básica dos profissionais da saúde como na formação continuada, têm pouca ou nenhuma oportunidade para aprender sobre saúde sexual e reprodutiva no contexto da deficiência (SOARES, 2008) repercutindo assim na não circulação da informação entre as PCD.

Uma vertente que surge dessa limitada oferta de informações é que as pessoas com deficiência estejam mais sujeitas a abusos sexuais, bem como a exposição a infecções sexualmente transmissíveis e mesmo ao preconceito que em um ciclo vicioso tente a ser um motivo de negação e omissão quanto a essa sexualidade. Nessa perspectiva é necessário resinificar os olhares para as transformações e adaptações necessárias a serem impressas na abordagem desse tema. 
Assim acredita-se que é necessário para aqueles que lidam e convivem com adolescentes com deficiência é preciso enfrentar os próprios estigmas quanto a sexualidade para que os espaços de interação e discussão da temática possam ser melhore explorados e vividos sem negações e com uma construção e condução progressiva e mais precisa da temática.

\section{CONSIDERAÇÕES FINAIS}

A questão da pessoa com deficiência vem ganhando e ocupando espaço, entretanto no que se refere à sexualidade, o tema ainda é envolto de estigmas. A sexualidade é muito mais ampla do que comumente é enxergada, proporciona um melhor desenvolvimento afetivo, auxilia a capacidade de se relacionar, melhora a autoestima e a adequação à sociedade. Assim, visualiza-se a importância de que a Sexualidade da Pessoa com Deficiência seja desmistificada e o cominho para o tema saia da marginalização, com a participação conjunta dos profissionais, envolvidos com esse público, incluindo o tema nos currículos de graduação. Embora o campo da sexualidade do adolescente com deficiência possua ainda um volume pequeno publicações, evidenciou-se que o mesmo apresenta uma curvatura ascendente em termos de volume de publicações principalmente após 2002, ano de publicação pelo Ministério da Saúde da Política Nacional de Saúde da Pessoa com Deficiência. É necessário um espaço de discussão pautado em elementos concretos, já estudados, analisados e estruturados a fim de oportunizar novos estudos e oportunidades.

\section{REFERÊNCIAS}

1. ALBUQUERQUE PP. Sexualidade e deficiência intelectual: um curso de capacitação para pais. Psicol. Argum., Curitiba, v.29, n.64, pp.109-119 jan./mar. 2011.

2. BASTOS OM, DESLANDES SF. Sexualidade e o adolescente com deficiência mental: uma revisão bibliográfica. Ciência \& Saúde Coletiva, Rio de Janeiro, v.10, n.2, p.389-397, 2005.

3. BASTOS OM, DESLANDES SF. Sexualidade e deficiência intelectual: narrativas de pais de adolescentes. Physis, Rio de Janeiro, v. 22, n. 3, p. 1031-1046, 2012.

4. BEZERRA CP, PAGLIUCA LMF. A vivência da sexualidade por adolescentes portadoras de deficiência visual. Rev. esc. enferm. USP, São Paulo, v. 44, n. 3, p. 578-583, Sept. 2010.

5. BRASIL. Portaria 1.060 de 5 de junho de 2002. Aprova a Política Nacional de Saúde da Pessoa Portadora de Deficiência.

6. BRASIL. Ministério da Saúde. Secretaria de Atenção à Saúde. Departamento de Ações Programáticas Estratégicas. Direitos sexuais e reprodutivos na integralidade da atenção à saúde de pessoas com deficiência. Brasília, DF, 2009. (Série B. Textos Básicos de Saúde).

7. CANO MAT, FERRIANI MGC, GOMES R. Sexualidade na adolescência: um estudo bibliográfico. Rev.latinoam. enfermagem, Ribeirão Preto, v. 8, n. 2, p. 18-24, abril 2000.

8. CAMARGO AMF, GUIMARÃES IRF, FRUET MSB et al. Sexualidade do adolescente. Pró-Posições, Campinas, v. 5, n. 3, p. 133-142, nov.1994.

9. CONOD L, SERVAIS L. Sexual life in subjects with intellectual disability. Salud pública México, v. 50, suppl.2, p. s230-s238, 2008.

10. COSTA MCO, LOPES CPA, DE SOUZA RP et al. Sexualidade na adolescência: desenvolvimento, vivências e propostas de intervenção. Jornal de Pediatria, Rio de Janeiro, v. 77, supl.2, p. S217-S224, 2001.

11. DAVIM RMB, GERMANO RM, MENEZES RM et al. Adolescente/Adolescência: Revisão Teórica Sobre Uma Fase Crítica Da Vida. Disponível em: http://www.revistarene.ufc.br/10.2/html/10_2_14.html.

12. DE PAULA AR, REGEN M, LOPES, P. "Sexualidade e Deficiência: Rompendo o Silêncio". Expressão \& Arte Editora, 2005

13. ESMAIL S, DARRY K, WALTER A et al. Attitudes and perceptions towards disability and sexuality. Disabil Rehabil. V. 32, n.14, p. 1148-55, 2010.

14. GEJER D, KLOURI C. O adolescente deficiente mental e sua sexualidade. Pediatria. Moderna, São Paulo, v. 38 , n. 8, p. 396-400, ago.2002. 
15. GIAMI A. O anjo e a fera: sexualidade, deficiência mental, instituição. São Paulo: Casa do Psicólogo, 2008.

16. GLAT R. Saúde sexual, Deficiência \& juventude em risco. Rio de Janeiro, março de 2004.

17. KAUFMAN M, SILVERBERG C, ODETTE F. The ultimate guide to sex and disability - for all of us who live with disabilities, chronic pain e illness. 2. ed. Califórnia/USA, Cleis Press, 2003.

18. LEITE MFMP, BORGES MS. Qualidade de vida do adolescente portador de cardiopatia: alguns aspectos práticos. Revista Oficial do Núcleo de Estudos da Saúde do adolescente, Rio de Janeiro, v. 4, n. 3, jul./set. 2007.

19. LEME CVD, CRUZ EMTN. Sexualidade e Síndrome de Down: uma visão dos pais. 2008.

20. LITTIG PMCB, CARDIA DR, REIS RB et al. Sexualidade na deficiência intelectual: uma análise das percepções de mães de adolescentes especiais. Rev. bras. educ. espec., Marília, v. 18, n. 3, p. 469-486, Sept. 2012.

21. LUIZ EC, KUBO OM. (2007). Percepções de jovens com Síndrome de Down sobre relacionar-se amorosamente. Revista Brasileira Educação. Especial, v.13, n. 2, pp. 219-238.

22. MAZZOTTA MJS, D'ANTINO MEF. Inclusão social de pessoas com deficiências e necessidades especiais: cultura, educação e lazer. Saude soc., São Paulo v. 20, n. 2, p. 377-389, June 2011.

23. MAIA ACB. Sexualidade e deficiências. São Paulo: Ed. Unesp, 2006.

24. MAIA ACB, CAMOSSA DA. Relatos de jovens deficientes mentais sobre a sexualidade através de diferentes estratégias. Paidéia, Ribeirão Preto, v.12, n.24, p.205-214, 2003.

25. MATOS M, CARNEIRO TF, JABLONSKI, B. Adolescência e relações amorosas: um estudo sobre jovens das camadas populares cariocas. Interação em Psicologia, jan./jun. 2005, 9(1), p. 21-33 1

26. MORALES AS, BATISTA CG. Compreensão da Sexualidade por Jovens com Diagnóstico de Deficiência Intelectual. Psicologia: Teoria e Pesquisa, Campinas, v. 26, n. 2, pp. 235-244, 2010.

27. MOURA GR, PEDRO ENR. Adolescentes Portadores De Deficiência Visual: Percepções Sobre Sexualidade. Rev Latino-am Enfermagem março-abril; v. 14, n. 2, p. 220-6, 2006.

28. NUNES CA. Desvendando a Sexualidade. $7^{\text {a }}$ ed. Campinas, SP: Ed. Papirus, 2005

29. ROMERO KT. O Conhecimento Das Adolescentes Sobre Questões Relacionadas Ao Sexo. Rev Assoc Med Bras 2007; 53(1): 14-9.

30. SCHLIEMANN AL, DUARTE DEST, GARCIA GS. et al. Sexualidade - adolescência - deficiência mental: um desafio a pensar. In: SIMPOSIO INTERNACIONAL DO ADOLESCENTE, 2, 2005, São Paulo. Proceedings online. 2005.

31. SOARES AHR, MOREIRA MCN, MONTEIRO LMC. Jovens portadores de deficiência: sexualidade e estigma. Ciência e Saúde coletiva, v. 13, n. 1, p. 185-198, 2008.

32. VIVARTA V. Mídia e Deficiência. Brasília: Agência de Notícias dos Direitos da Infância; Fundação Banco do Brasil. 2003. 